\title{
Gradhiva
}

GRADHIV

Revue d'anthropologie et d'histoire des arts

$4 \mid 2006$

Le commerce des cultures

\section{Le commerce de la culture, la médecine rituelle et le Coca-Cola}

Marcelo Fiorini et Christopher Ball

Traducteur : Olivier Allard

OpenEdition

Journals

Édition électronique

URL : http://journals.openedition.org/gradhiva/605

DOI : $10.4000 /$ gradhiva. 605

ISSN : $1760-849 x$

Éditeur

Musée du quai Branly Jacques Chirac

Édition imprimée

Date de publication : 1 novembre 2006

Pagination : $97-113$

ISBN : 2-915133-44-1

ISSN : 0764-8928

\section{Référence électronique}

Marcelo Fiorini et Christopher Ball, «Le commerce de la culture, la médecine rituelle et le Coca-Cola », Gradhiva [En ligne], 4 | 2006, mis en ligne le 15 novembre 2009, consulté le 10 décembre 2020. URL: http://journals.openedition.org/gradhiva/605 ; DOI : https://doi.org/10.4000/gradhiva.605

Ce document a été généré automatiquement le 10 décembre 2020.

(C) musée du quai Branly 


\title{
Le commerce de la culture, la médecine rituelle et le Coca-Cola
}

\author{
Marcelo Fiorini et Christopher Ball \\ Traduction : Olivier Allard
}

1 Cet article explore les conditions complexes de production et de représentation d'un rituel amazonien, la danse des masques Atujuwá des Wauja du Brésil, exécutée à l'occasion d'une invitation rémunérée à Montpellier en juillet 2005. Les deux mécènes de cet événement étaient le musée du quai Branly alors naissant, c'est-à-dire le prestigieux musée ethnographique de Paris, et le festival de musiques du monde de Radio France et Montpellier. Il faisait partie d'un ensemble de spectacles et d'expositions à travers le pays qui marquèrent en 2005 l'année du Brésil en France.

2 Les Wauja venus en France - dix-sept hommes adultes - font partie d'une communauté d'approximativement trois cent quarante personnes qui résident pour la plupart dans un unique village du parc indigène du Xingu (PIX), dans le Mato Grosso (Brésil). Ils parlent une langue de la famille arawak, et sont les gardiens d'une riche tradition culturelle, à la fois matérielle et rituelle, comprenant notamment la danse et la musique verbale et instrumentale (Mello 2005), ainsi que les céramiques en terre et les masques en fibre, qui se caractérisent également par une iconographie complexe (Coelho 1993, Barcelos Neto 2001, 2002). Si les Wauja avaient été invités à donner une représentation en France, c'était essentiellement en raison de l'aspect frappant et de la qualité esthétique des masques Atujuwá, les plus grands dans le panthéon des masques wauja, dont la partie supérieure est constituée de disques qui peuvent atteindre 2,5 mètres de diamètre.

3 L'article présenté ici ne traite pas précisément de l'exécution d'un rituel wauja traditionnel. Aristóteles Barcelos Neto, l'anthropologue responsable de la conception et de la direction scientifique du spectacle montpelliérain, remarque dans un avertissement introductif du programme officiel de l'événement que ce qui a été montré en France n'était « pas le rituel. Celui-ci s'est déroulé au village wauja à la fin du mois de mars 2005. C'est à cette occasion, dans un espace-temps très différent de celui de Montpellier, 
qu'eurent lieu la fabrication des masques et la distribution d'aliments et de dons aux danseurs ». (Barcelos Neto $2005: 15)$

4 À la différence de Barcelos Neto, nous soutenons que le rituel effectué dans le village wauja en mars 2005 était déjà un événement transformé, doté d'une signification originale dans un contexte totalement nouveau de relations d'échanges : ce n'était donc " pas le rituel », là non plus, si on entend par ce terme la pratique wauja traditionnelle de thérapie rituelle par la danse des masques Atujuwá.

Mais si aucun de ces objets culturels n'est le rituel, peut-être chacun d'entre eux est-il toujours un rituel, d'une certaine manière. Un rituel précisément inscrit dans des relations sociales d'échanges dont la signification se situe dans une dimension spatiotemporelle transformée. Nous prétendons toutefois ici que les rituels ne peuvent pas être uniquement compris comme des scripts conventionnels utilisés à titre de ressource et mis en scène dans des contextes sociaux particuliers. Au contraire, ce sont des objets culturels complexes, dont l'exécution est le véhicule d'une intentionnalité [agency] sociale qui redéfinit constamment les relations sociales, notamment lorsqu'il s'agit d'un contact interculturel ou interethnique.

6 En ce sens, cet aspect passé sous silence du rituel - être un objet culturel capable d'être importé pour un spectacle monté en France - correspond précisément à la reconnaissance, la négociation et l'interprétation, selon des régimes idéologiques particuliers, de relations sociales et de champs culturels nouvellement établis, notamment ceux qui prévalent entre les anthropologues, leurs sujets d'étude, les mécènes et les spectateurs éventuels de l'événement culturel. Nous nous concentrons ici sur les conditions de spatialisation de ces relations sociales, ainsi que sur l'articulation d'idéologies particulières pour les interpréter. Le déroulement de tels instants de contact interculturel dépend en grande partie des structures socioculturelles et sémiotiques en place. Dans notre cas, il s'agit tout d'abord de l'appareil institutionnel qui permet d'exécuter avec succès la danse des masques wauja, selon une idéologie inhérente d'intentionnalité sociale qui est à la fois implicite et articulée dans son exécution. Il s'agit ensuite de la posture des mécènes français, en particulier de la manière dont leurs théories institutionnelles de la représentation ou de la propriété culturelle entrent en conflit ou en harmonie avec l'assimilation idéologique récurrente des objets culturels à des marchandises évaluées en termes d'authenticité.

7 Nous soutenons précisément que les différences d'interprétation de ces processus parmi les exécutants du rituel wauja en France font partie d'un processus plus vaste qui touche la société wauja, où des modes nouveaux de circulation spatiotemporelle sont liés à des idéologies changeantes de la santé et de la maladie, de la cosmologie, de l'échange et de la culture mise en scène, qui est désormais traitée comme un objet.

\section{Le commerce de la culture}

8 L'importation et la représentation publique de cérémonies rituelles venues du bout du monde est une longue tradition dans l'histoire du colonialisme et a une histoire particulière dans le cas du Brésil et de la France. Les spectacles coloniaux entraînaient une conception mythifiée et ethnocentrique des autres cultures, et satisfaisaient à un désir latent d'exotisme. Il ne s'agissait pas de tentatives réelles de décrire ces cultures dans leurs propres termes. Évidemment, les organisateurs du spectacle de danse Atujuwá 
en France, et en particulier le musée du quai Branly, ont fait de grands efforts pour se distinguer des événements coloniaux ethnocentriques: ils ont ainsi fourni des informations ethnographiques concernant les Wauja, leur cosmologie, la danse des masques, le parc du Xingu, l'Amazonie et l'histoire de l'anthropologie chez les Amérindiens. Des représentants du musée nous ont déclaré sur place que la décision de montrer la danse avec les masques, c'est-

à-dire d'importer le contexte avec l'artefact, était une caractéristique originale de la perspective ethnologique du musée. Elle fournissait en effet le fondement ethnographique de la cérémonie rituelle, en essayant d'éviter la décontextualisation de tels objets culturels.

9 L'anthropologue brésilien Aristóteles Barcelos Neto, qui a écrit sur l'iconographie, la mythologie, la cosmologie et les connexions entre chamanisme et pratique rituelle chez les Wauja, a négocié la vente de dix-sept masques en fibre de palme au musée du quai Branly et a organisé, en coordination avec ce dernier, le spectacle de leur présentation à Montpellier. Ce n'est pas la première fois que des masques Atujuwá sont faits sur commande (Barcelos Neto 2004). Au cours des dernières années, des danses ont été financées et organisées dans le village wauja avec l'intention de "donner » les masques aux collections ethnographiques de musées. Cependant, dans le long processus qui a conduit au voyage en France, la demande d'une fabrication de masques destinés à la vente a créé des controverses dans la communauté wauja. Si de nombreux Wauja étaient disposés à voyager et à être payés en argent, d'autres se montraient plus réticents à cet égard dans la mesure où l'exécution de la danse des masques hors du village, dans un pays lointain dont ils n'étaient pas familiers, représentait une expérience inédite.

L'expérience qu'ont les Wauja de ce type de commerce de la culture doit être située dans un processus plus vaste de spatialisation culturelle, qui ne doit pas nécessairement être interprété comme une pure appropriation par des agents externes grâce à l'hégémonie capitaliste. Elle ne doit pas non plus être vue comme la réification ou la marchandisation d'objets culturels

- ce qui en ferait une conception exclusivement importée plutôt qu'autochtone. Selon Graham (2005), les efforts en ce sens des Xavante voisins ne constituent aucunement une relation d'échange direct. À la différence des Xavante, les Wauja semblent conceptualiser clairement les projets interculturels, notamment ceux qui présentent la « culture » à des spectateurs non indigènes, comme des relations d'échange direct - une idéologie qui masque en fait que ces dernières se caractérisent par un dense tissu de médiations. Nous suggérons que cet aspect du commerce de la culture n'est pas un indice de corruption, c'est-à-dire ici de marchandisation, mais une forme de continuité culturelle dans la mesure où un système compétitif fondé sur l'échange et impliquant la circulation de divers «objets » culturels est une caractéristique centrale de l'organisation du HautXingu $^{1}$ (voir Agostinho 1974, Gregor 1977, Viveiros de Castro 1977).

11 Les peuples indigènes du Xingu, dont font partie les Wauja, appartiennent à une aire culturelle où sont présents onze groupes ethniques différents, parlant six langues distinctes, et se conformant à un accord de paix et de partage dans un système d'échange régional complexe auquel participent tous les villages du sud du PIX. Ils ont réussi à créer avec la société brésilienne environnante une forme de contact interculturel à travers laquelle ils contrôlent l'entrée des biens et des pratiques étrangères dans leur région, en adoptant largement ce qui leur semble bénéfique sans s'interdire les expérimentations. Ils ont également obtenu des médias qu'ils leur accordent une place importante tout en 
donnant d'eux une image positive. En effet, ils ont un don naturel pour représenter la culture et un sens aigu de la politique de l'identité. Ce n'est pas une coïncidence si les groupes indigènes du Xingu sont devenus la « carte postale » de l'indigénéité brésilienne (Lagrou 2002: 19).

La réification des objets culturels en tant qu'indices de l'identité locale a une histoire qui précède la marchandisation récente de rituels comme le Kwarup, la cérémonie annuelle de fin de deuil qui est devenue un emblème de la culture du Haut-Xingu ${ }^{2}$. Le système régional d'échange rituel lui-même a conduit à de tels processus. Depuis quelque temps déjà, le Kwarup est un événement public auquel assistent souvent de nombreux visiteurs " payants». Ces dernières années, des groupes du Xingu ont également commencé à organiser des Kwarup en l'honneur de Brésiliens importants comme Orlando Villas-Boas, l'agent gouvernemental dont le travail a inspiré la création du PIX. Plus de cent vingt non-indigènes ont assisté en 2003 à ce Kwarup dans le village yawalapiti, dont des journalistes de Globo TV, Radio-TV Bandeirantes, Folha de São Paulo, Jornal do Brasil, National Geographic, du Washington Post et du New York Times ${ }^{3}$. En 2004, sous les auspices de Globo TV, un leader kamayura a organisé un Kwarup en mémoire du journaliste Roberto Marinho, qui était le président du groupe de médias le plus puissant d'Amérique latine.

On pourrait écarter de tels événements en y voyant le produit d'une simple appropriation culturelle. Mais on s'aveuglerait alors sur la manière dont les peuples du Haut-Xingu les ont utilisés pour créer de nouveaux réseaux sociaux en établissant des alliances avec d'autres peuples indigènes, des organisations non gouvernementales ou divers sympathisants de l'autre bout du monde. De telles alliances les ont libérés d'une dépendance antérieure à l'égard de la Fondation Nationale de l'Indien (FUNAI) en détruisant les barrières paternalistes de pouvoir et de domination néocoloniale en place depuis soixante ans. De plus, puisque de tels choix émanent du Haut-Xingu lui-même, principalement à travers des décisions prises collectivement par des leaders locaux déjà largement exposés au monde extérieur, nous pensons que les anthropologues ne devraient pas fuir la participation à de tels processus ou faire semblant de se contenter de les étudier 4 . Les alliances entre ethnies du Xingu et agents extérieurs, qui puisent dans le savoir anthropologique, ont marqué la politique locale et nationale dès le début du travail des anthropologues dans la région ${ }^{5}$. La dimension sociale de telles transformations culturelles doit encore être évaluée, mais il est clair que les peuples du Xingu se sont aujourd'hui engagés dans un projet de production interculturelle à l'échelle nationale et internationale qui peut être caractérisé, dans les termes de Myers (2003:350), comme un exemple de « (re)fabrication de la culture ».

\section{L'intentionnalité rituelle en action}

14 Afin de voir le lien entre échange et rituel, nous abordons maintenant l'action rituelle comme pratique thérapeutique chez les Wauja, en parcourant la chaîne d'événements qui aligne les individus dans le cycle allant de la maladie à l'exécution du rituel et au rétablissement de la santé à travers la danse des masques Atujuwá (voir aussi Barcelos Neto 2004 : 142-147, pour une explication convaincante et plus détaillée de ce processus).

15 La maladie et la souffrance humaine sont souvent attribuées chez les Wauja au contact avec les apapaatai, ou esprits-monstres ${ }^{6}$. Ainsi, l'action humaine organisée et spécialisée a souvent pour but de rectifier les effets d'un tel contact. Les victimes peuvent voir 
différents types d'esprits-monstres en rêves, notamment les Atujuwá, et on peut demander aux chamanes d'interpréter de tels rêves pour en évaluer la cause et les effets possibles, puis de débuter le traitement. À cette étape, les chamanes soufflent de la fumée de tabac sur la victime afin d'aider le diagnostic et le traitement. Pendant que le traitement chamanique spécialisé se poursuit, l'éloignement de l'esprit-monstre peut être facilité par un contrat entre des membres de la famille de la victime et des membres spécifiques de la communauté, auxquels est assigné le rôle de kawoká-mona (représentant de l'esprit-monstre). Les parents de la victime offrent de la nourriture sur la place du village, nourrissant ces représentants et par là même l'esprit-monstre pathogène. Ces derniers agissent comme les esprits-monstres qu'ils représentent. Parés de coiffes emblématiques mais modestes, ils vont en groupe

du centre du village jusqu'au hamac de la victime en disant par exemple: " Je suis ton esprit protecteur, je suis Atujuwá. » Ils soufflent ensuite de la fumée de tabac sur la victime et partent, figurant ainsi la sortie de l'esprit-monstre.

En reprenant connaissance, la victime devient un propriétaire ou maitre, owekeho, de l'esprit-monstre pathogène, qui est désormais son esprit protecteur

(okawokala). C'est également lui qui détient le droit et la responsabilité d'être le mécène du rituel. Si un maître d'Atujuwá a les ressources matérielles suffisantes pour célébrer son kawoká, il peut décider de financer le rituel de l'esprit-monstre Atujuwá à travers un " paiement» (sous forme d'échange) aux représentants de ce dernier (les kawoká-mona), qui vont alors organiser, préparer et exécuter les différentes phases du rituel. Celles-ci vont de la danse à la musique de flûte et au chant en fonction de l'identité des espritsmonstres, mais requièrent toujours que le corps soit peint et paré de manière élaborée. $\mathrm{Si}$ la victime est toujours souffrante, tout ceci peut être entrepris comme une action d'urgence afin de restaurer des relations sociospatiales appropriées entre les humains et les apapaatai, en apaisant et en congédiant ces derniers. Mais la victime peut aussi financer un rituel longtemps après sa rémission, à la suite d'une maladie isolée ou d'une série de maladies: elle a accumulé un stock d'esprits-monstres qu'elle doit honorer à travers la cérémonie, dont l'enjeu fonctionnel est de maintenir la distance sociospatiale appropriée entre les humains et les esprits-monstres.

17 Selon la théorie wauja de la maladie, les attaques d'esprits-monstres ou de dards magiques entrainent

la séparation entre l'âme de la victime et sa personne. Lorsque quelqu'un désire certaines nourritures qu'il ne peut pas obtenir, il entre dans un état de witsixu, de faiblesse psychique et spirituelle, où il est particulièrement vulnérable aux attaques (Barcelos Neto 2002). Les attaques des esprits-monstres sont le résultat d'une proximité exacerbée entre ces derniers et les humains, par laquelle le corps des esprits-monstres touche les humains et laisse en eux la cause de la maladie (Barcelos Neto 2004). Il ne s'agit pas nécessairement d'une action mal intentionnée de la part des esprits-monstres, mais plutôt d'une question de frontières poreuses entre des êtres sociaux appartenant à différents ordres de l'univers, ce qui rend dangereuse une proximité non maîtrisée. Pour les Wauja, contrôler et satisfaire le désir sont les conditions nécessaires de la bonne santé, contribuant à maintenant les esprits-monstres à leur place appropriée. L'exécution du rituel Atujuwá implique donc un certain risque, dans la mesure où il entraîne une proximité avec les esprits-monstres.

18 Une caractéristique du rituel Atujuwá est qu'aucun moment défini de la célébration ne symbolise précisément la cure. L'efficacité du rituel repose apparemment plutôt sur les 
actions elles-mêmes, au moment où toutes les ressources rassemblées par le maitre ont été épuisées et où la danse atteint son paroxysme. Selon Barcelos Neto (2004), il s'agit également de l'étape la plus importante et la plus agréable esthétiquement de la danse des masques. Les représentants des esprits-monstres doivent contenter l'Atujuwá à travers l'exécution du rituel, afin qu'il parte à nouveau. Dans de nombreux cas, après la production et l'exécution d'un rituel, les représentants donnent des marques de reconnaissance pour la nourriture qu'ils ont reçue durant le processus rituel : des colliers, des céramiques, etc. De tels contre-paiements sont décrits comme visant en même temps le maître et son kawoká, son esprit-monstre protecteur.

19 Si l'on détaille plus précisément les relations sémiotiques qui entrent en jeu dans la cure rituelle des Wauja, on constate que la victime ou le patient, puis les chamanes, les représentants du kawoká-mona ${ }^{7}$ et le maitre du rituel personnifient tous les espritsmonstres. De telles identifications sont révélées en pratique par l'action sociale et rituelle, ainsi que par l'échange d'objets culturels comme la nourriture, le tabac, les boissons sucrées, ou la danse des masques elle-même ${ }^{8}$. Il serait risqué de décrire l'activité des représentants comme une pure question de « représentation »- que ce soit dans la cure, la parole, le chant, la musique des flûtes, la danse, les masques ou la peinture corporelle. Au contraire, c'est précisément l'incorporation des actions rituelles dans les relations sociales - par laquelle les représentants "deviennent» les esprits-monstres, produisent et échangent des objets culturels avec le patient/maitre - qui donne à ces représentants des pouvoir curatifs et rend effective leur exécution du rituel. De plus, c'est la logique des interactions réciproques qui est en réalité le moteur du rituel. L'essentiel est que se glisser dans une position sociale requiert l'exécution d'actions concomitantes, actions qui sont uniquement valorisées ou considérées comme effectives si elles produisent un certain état du monde : un traitement chamanique qui fait d'un individu le maitre d'un esprit-monstre protecteur, une prestation rituelle qui rend possible l'activité rituelle des représentants des esprits-monstres, un échange jusqu'à l'épuisement des ressources, une danse des masques esthétiquement plaisante, et finalement un retour des biens, ou des « choses » de l'esprit, à l'esprit-monstre protecteur.

Chacune de ces étapes implique une identification séparée avec l'Atujuwá, mais l'identification, à l'origine nuisible, se fait progressivement inoffensive. L'esprit-monstre pathogène devient un protecteur, le patient un maître, les chamanes (et d'autres) des représentants (kawoká-mona - c'est-à-dire des danseurs), et les Atujuwá retrouvent leur dimension spatiotemporelle inoffensive. En ce sens, le rituel des Wauja semble se définir, en suivant l'argumentation de Houseman et Severi (1994:185), par les actions en ellesmêmes, sans que celles-ci soient interprétées comme des représentations de significations ou de symboles sous-jacents. Selon nous, l'ordre de telles actions et leurs transformations seraient également importants pour rendre effective la danse des masques des Wauja.

21 Mais quelles sont les mesures protectrices qui existent dans la société wauja lorsqu'un maître échoue à maintenir une relation appropriée avec les représentants des espritsmonstres (et vice-versa) ? En manquant à son devoir de nourrir les exécutants du rituel, par exemple, le maître peut faire souffrir et mécontenter les esprits-monstres, ce qui risque de donner une tournure indésirable à leur relation. C'est exactement cela qui a produit la maladie en premier lieu. De plus, comme les rôles d'Atujuwá et de représentant d'esprit-monstre sont vus comme identiques, les représentants en pâtissent également. Retournons maintenant au contexte du spectacle de danse des masques Atujuwá en 
France, et à celui de son événement préparatoire dans le village wauja. Nous comprendrons ainsi comment ces deux célébrations peuvent nous donner des indices de la manière dont non seulement le rituel, mais aussi certaines de ses parties peuvent être objectivés afin de transformer ou d'affecter les relations d'échange ou l'efficacité des résultats attendus. Mais il convient tout d'abord de faire un large détour à travers le mythe pour montrer comment il est possible que le rituel Atujuwá lui-même reflète une relation ratée qui a transformé le monde wauja.

\section{Achèvements mythiques}

Emilienne Ireland, une anthropologue américaine qui a étudié les Wauja dans les années 1980, a fait l'hypothèse que le mythe expliquant l'apparition des Atujuwá fait allusion à l'arrivée historique de l'explorateur allemand Karl von den Steinen, que l'on reconnaît être le premier Blanc à pénétrer la région du Haut-Xingu en 1884 et 1887 (Ireland 1988 : 169 et 174). Comme toutes les créatures mythologiques, hybrides d'humanité et d'animalité ou de phénomènes physiques, l'Atujuwá de ce mythe est également un hybride - mais qui apparaît bien étrange (Ireland 1988 : 168 [1'auteur reprend ici les dires des Wauja]) :

«[... les Atujuwá] étaient squelettiques, poilus, blancs de peau... Leur peau était couverte de petits grains de beauté et de boutons bruns. Des poils poussaient sur leur visage. Leurs ceintures n'étaient pas des ceintures de coquillage comme les nôtres, mais des serpents qui avaient été noués autour de leurs corps. Même leurs ventres étaient très poilus. Et leurs jambes étaient couvertes de fibre de paille. Audessus de la ceinture, ils ressemblaient à des hommes, mais en dessous, on ne pouvait pas voir de peau, seulement de la paille. Ils remplissaient l'air de leur danger et de leur pouvoir ; rien que de les toucher brûlait comme du feu... »

Ireland reconnaît que les Atujuwá sont, selon les Wauja, des esprits-monstres et non des Blancs, et que le mythe qui marque leur apparition traite de l'extinction d'un groupe voisin, les Kustenau, et non de l'arrivée des Blancs. Selon elle, cependant, la « ressemblance frappante» des êtres mythiques peints comme " poilus, barbus, blancs", ou comme des humanoïdes avec "taches de rousseur et grains de beauté", et "un vêtement ressemblant à un pantalon en dessous de la ceinture » - d'autant plus que leur apparition dans le récit mythique est associée avec l'« extinction » des Kustenau, qui ont été effectivement rayés de la carte immédiatement après le contact par les «maladies des Blancs »-, tous ces éléments devraient être une preuve suffisante pour affirmer que l' Atujuwá est une « représentation » de la conception wauja du Blanc (1988: 169).

Nous voudrions radicaliser l'hypothèse exposée dans la section précédente en affirmant que les mythes, comme les rituels, peuvent être également conçus comme des mises en actes plutôt que comme de simples processus ou encodages symboliques. En ce sens, ce qu'avance le mythe de l'Atujuwá - s'il s'agit effectivement d'une représentation de l'arrivée du Blanc-, c'est avant tout une interprétation de la position que

le Blanc en vient à occuper dans la conscience wauja, comme un être apparenté à une série d'esprits-monstres ancestraux que l'on doit apaiser par de la nourriture et des prestations afin de transformer ces êtres potentiellement dangereux, présages de chaos, de maladie et de mort, en une influence bénéfique. On peut se rappeler le rituel où un esprit-monstre est transformé en protecteur et devient un bienfaiteur qui peut même contribuer à la cure wauja. Si l'on suit cette hypothèse, il faut se demander s'il existe des éléments qui confirment l'idée que le Blanc était effectivement considéré - et pas 
seulement dépeint dans le mythe - par les Wauja comme un esprit-monstre protecteur ancestral.

Puisque les Wauja reconnaissent que les Atujuwá sont des esprits de tornades et de trombes qui représentent une force naturelle destructrice, on pourrait dire que le Blanc est juste un autre "quelque chose ", un être qui s'interpose entre un objet, l'Atujuwá, et le concept d'esprit-monstre. De ce point de vue, il importe peu de savoir qui est arrivé en premier. En revanche, c'est l'intentionnalité de quelqu'un qui détermine sa relation avec les Atujuwá, de même que sa survie ou sa chute, comme semblent l'impliquer les Wauja. Dans le mythe, les Kustenau dégénèrent dans une épidémie dévastatrice de sorcellerie après s'être approprié des objets "fétiches" issus des corps des Atujuwá. C'est donc l'interaction des Kustenau avec les Atujuwá (Blancs) qui provoque leur fin, à la fois dans le mythe et dans l'histoire.

Il est remarquable que Steinen ait été surpris par l'augmentation considérable du nombre de masques qu'il a vus dans le Haut-Xingu lors de son second voyage dans la région en 1887, en particulier de ceux représentant des visages monstrueux d'apparence (sur)humaine, en bois et en paille (1940 [1894] : 380). Cependant, les grands masques Atujuwá que nous voyons aujourd'hui, surmontés de larges disques de plus de 2,5 mètres de diamètre, n'étaient pas encore vraiment apparus 9 . On pourrait ainsi spéculer que les parallèles ou les similarités entre les masques Atujuwá et Karl von den Steinen et les autres membres de son expédition vont bien au-delà de la profusion vestimentaire qui les caractérise en commun et touchent d'autres éléments présents dans le mythe d'apparition des Atujuwá, que l'on peut rapprocher des photographies de l'expédition. Ainsi, les Atujuwá portent des ceintures qui semblent être des serpents noués autour de leurs corps, ce qui évoque les larges ceintures portées de manière lâche par les membres de l'expédition, de même que leurs chapeaux de Panama ressemblent à de larges disques surmontant leurs têtes et leurs corps, exactement comme les masques d'aujourd'hui ${ }^{10}$.

Nous ne soutenons pas que les Blancs sont personnifiés dans le rituel wauja, ni que les masques en sont une exacte représentation. Nous suggérons que la quantité même et la place centrale accordée à l'iconographie et l'esthétique de la représentation des espritsmonstres dans le rituel wauja témoignent de l'importance donnée à la forme et aux détails figuratifs, ainsi qu'à toutes les allusions qui en découlent. Nous voyons la célébration montpelliéraine comme étant un prolongement de cette fabrique ritualisée du mythe. En France, les masques et la danse sont devenus un indice de la relation entre les Wauja et les organisateurs de l'événement, tout en incarnant des signes complexes, ouverts à l'interprétation, et ainsi l'exécution de la danse des masques pourrait être vue comme une nouvelle mise en acte du mythe des Atujuwá. Non pas comme le retour en Europe d'un fétiche de Steinen approprié par les Kustenau, mais peut-être comme la forme par laquelle les Wauja ont réussi à apprivoiser un être monstrueux qui remplissait l'air de son danger et de son pouvoir, et ainsi à remplacer la terreur par la beauté.

\section{De Piyulaga à Montpellier}

Les deux dernières danses de masques Atujuwá exécutées à Piyulaga, le village des Wauja, ont été considérablement altérées par la présence de photographes et d'équipes de tournage. En mars 2005, trois personnes du Laboratoire d'anthropologie de l'image et du son (LISA) de l'université de São Paulo (USP), dont Barcelos Neto était le conseiller/ directeur, sont allées dans le village afin de filmer la danse des masques. Un des auteurs 
de cet article (Ball) était dans le village wauja juste avant l'événement, alors que l'autre (Fiorini) y est arrivé un jour après la première équipe, afin de filmer également l'exécution du rituel, de manière indépendante ${ }^{11}$.

Barcelos Neto (2005: 12) raconte que, dans le « rituel » qui s'est produit dans le village wauja à la fin de mars 2005, un arrangement pour son mécénat par procuration fut trouvé avec la communauté : un " groupe de maîtres, avec à leur tête un chamane visionnairedevin ", allait s'occuper de l'Atujuwá pour le compte des institutions françaises qui le finançaient. Il était cependant clair pour les villageois, pendant les préparations comme la fabrication ou la peinture des masques, qu'aucun individu n'occupait le rôle de mécène du rituel. En effet, certaines personnes spéculaient que l'Atujuwá pouvait frapper un individu et lui faire occuper le rôle de maitre à cette étape. Certains avançaient également que l'Atujuwá pouvait approcher un individu qui pourrait ou devrait être le maître, mais ne faisait pas les prestations appropriées ${ }^{12}$. De plus, d'autres conditions rituelles à la fabrication et à l'exposition des masques n'étaient pas remplies. Pour commencer, bien que le rituel Atujuwá ne soit pas saisonnier, il ne s'effectue jamais pendant la saison humide, parce qu'il n'y a pas assez de nourriture à cette époque de l'année pour alimenter les esprits-monstres voraces. Cette situation fut aggravée par le fait que l'équipe de tournage de l'USP arriva au village presque sans nourriture : plusieurs exécutants de la danse se demandèrent alors si le rituel pouvait se poursuivre dans un tel contexte de disette ${ }^{13}$.

Bien qu'ayant commencé sous de mauvais auspices, le projet gagna finalement le soutien de toutes les fractions du village : après tout, dix-sept hommes avaient été choisis pour représenter les Wauja, et leur participation à l'événement en France allait apporter des bénéfices à l'ensemble de la communauté. Mais lorsqu'un enfant tomba malade, les chamanes commencèrent immédiatement à craindre que les esprits-monstres n'en soient la cause. Ensuite, alors que

les danses étaient déjà entamées, un des chamanes appelés pour réaliser la cure se mit à courir en état de transe, traversant plusieurs fois la place du village. L'exécution du rituel s'arrêta immédiatement. L'appréhension des participants wauja allait croissante : certains soulignèrent le manque de nourriture et l'époque inappropriée pour réaliser ce rituel, de même que l'absence d'un maître manifeste du rituel, ou encore le fait même que les Wauja étaient en train de se mêler d'une célébration potentiellement dangereuse à la demande des équipes de tournage.

$31 \mathrm{Au}$ moment où la danse aurait dû s'accélérer et devenir le seul centre d'attention de chacun, l'attitude de la plupart des exécutants allait du manque d'enthousiasme à une posture volontairement nonchalante. Cependant, leur participation était de la plus haute importance, puisque l'anthropologue/directeur voulait absolument que le cercle d'une chaude couleur jaune soit peint sur la surface des masques avant que ces derniers ne soient envoyés en France. On peut penser que, sans ce jaune d'or, les masques ne seraient apparus que peints à $90 \%$ pour quiconque les aurait déjà vus entièrement finis. De plus, les équipes de tournage attendaient toujours le paroxysme de la danse, lorsque tous les Atujuwá sortiraient en même temps de la maison des hommes et tourneraient autour du village. La situation s'arrangea finalement quand l'état de l'enfant s'améliora, et qu'un bol de piments rouges fut apporté à la maison des hommes et offert comme paiement aux esprits-monstres. Ceci sembla apaiser les représentants des Atujuwá, puisque le final de la danse des masques eut lieu à la fin de l'après-midi, « entre chien et loup » (le meilleur moment pour photographier ou filmer), pour satisfaire les équipes de tournage présentes, 
et l'exécution, qui était à la fois une répétition et un scénario de film, s'effectua brillamment.

\section{La nourriture sympathique : eau, escargots et Marlboro}

En arrivant à Montpellier, les Wauja remarquèrent que l'eau que l'on avait achetée pour eux leur paraissait salée, en comparaison de l'eau douce du Haut-Xingu. Mais puisqu'elle avait été acquise à l'avance et en grande quantité, les organisateurs ne firent aucun effort pour l'échanger. Une autre difficulté apparut le matin même de l'arrivée des Wauja: aucun ticket n'avait été fourni pour leur petit-déjeuner et, sans argent, ils ne pouvaient pas manger dans la cafétéria du campus universitaire où ils avaient été logés. Lorsque le déjeuner arriva, les Wauja découvrirent avec stupéfaction que les portions de la cafétéria étaient incroyablement petites selon leurs critères (ou même ceux des Brésiliens). De plus, ils eurent la malchance d'avoir des spaghetti aux pétoncles sur le menu de ce jour, et, comme ils avaient entendu dire avant leur voyage que les Français mangeaient des escargots, la plupart des hommes ne touchèrent pas leur assiette - à l'exception d'un d'entre eux qui fit l'effort d'avaler une petite bouchée - alors même qu'ils mouraient de faim $^{14}$. Les Wauja commencèrent à penser qu'ils n'étaient pas traités avec bienveillance, ni même nourris convenablement alors qu'il s'agit de deux des formes basiques d'hospitalité qu'ils étaient en droit d'attendre. En même temps, les Wauja craignaient sérieusement que les Atujuwá ne soient aussi peu satisfaits qu'ils l'étaient eux-mêmes, et que certains tombent malades très rapidement. Tout ceci fut exprimé très succinctement par un des participants ${ }^{15}$ :

« Vous savez que l'Atujuwá existe... Nous faisons l'Atujuwá [son masque] et nous le célébrons ${ }^{16}$. Il nous fait mal, il nous tue. C'est pourquoi les chamanes fument du tabac, pour voir l'Atujuwá. Il peut nous faire mal, nous faire saigner du nez, par exemple. Pour cette raison, nous célébrons l'Atujuwá, afin qu'il nous laisse tranquille, qu'il nous laisse en paix et en bonne santé. L'Atujuwá peut bien nous traiter, et c'est pourquoi on doit le faire/célébrer. Vous devriez lui préparer de la nourriture, beaucoup de nourriture : du poisson, du ragoût, de la boisson. Vous lui en donnez, et l'Atujuwá l'apprécie, il vous laisse en paix. »

L'autre chose qui manquait aux Wauja était le tabac, non seulement comme élément important des préparations du rituel, mais aussi comme véritable nécessité pour la plupart des hommes, et en particulier pour les chamanes venus en France. En raison de l'absence de tabac en vrac, la plupart des hommes demandèrent des Marlboro. Les Wauja ont l'habitude de fumer des cigarettes à Canarana, la ville frontière aux abords du PIX, mais n'avaient pas les moyens d'en acheter à Montpellier. Cependant, leur demande de cigarettes était perçue par les organisateurs comme un luxe et non comme une nécessité. Peu après leur arrivée en France, les exécutants wauja étiquetèrent rapidement un des organisateurs comme le maître du rituel, et avaient une série d'attentes précises au sujet de la manière dont cet acteur devrait maintenir une relation sociale appropriée avec eux. Un chamane les exprima clairement :

« Dis-lui [le chercheur] que je l'ai vu [l'Atujuwá]. Je l'ai vu plus tôt [en rêve $\left.{ }^{17}\right]$. Il n'est pas venu et n'a pas fumé de tabac plus tôt, hier soir. Il n'a pas fumé de tabac. C'est très mauvais pour l'esprit-monstre. Ensuite, on nous a acheté un peu de tabac, juste un petit peu. On nous a acheté du tabac et l'esprit-monstre a fumé. Il l'a apprécié. C'était bon pour lui. Tu vois, il ne s'approchait plus de nous. L'esprit-monstre ne s'approchait plus de nous. Il avait fumé du tabac. Alors, ensuite "achète-nous du 
tabac", a-t-on dit à cette personne qui a programmé le rituel, qui nous a invités. Nous avons demandé à cette personne "achète-nous du tabac" pour que les espritsmonstres puissent fumer, pour qu'ils ne nous fassent pas de mal, pour qu'on puisse bien vivre. Cet endroit est très loin de chez nous. » eux) et un don pour la communauté, dont le montant avait été négocié. Mais, en plus de ce paiement en argent, ils attendaient des quantités copieuses de tabac, de nourriture et de boissons (sucrées, sans alcool). Dans le rituel de l'Atujuwá, le maître en fournit aux représentants/exécutants jusqu'à l'épuisement de ses propres réserves et de celles qu'il peut rassembler en demandant à ses parents de contribuer. Les organisateurs français paraissaient mal comprendre la nécessité rituelle de ce qui pouvait leur sembler d'incessantes requêtes de la part d'Indiens voraces et peu authentiques qui voulaient plus de nourriture et de Marlboro ${ }^{18}$.

\section{La politique des ondes}

Alors que les Wauja avaient exprimé aux organisateurs de l'événement montpelliérain toutes leurs insatisfactions concernant les conditions de leur séjour en France, eut lieu un incident surprenant. Il nous révéla à quel point les relations établies à travers le mécénat du rituel exécuté en France étaient devenues pour les Wauja un moyen de retrouver le sentiment de leur position d'agents intentionnels et de leur capacité à influencer ou à transformer la situation dans laquelle ils s'étaient retrouvés.

Dans une interview radiophonique qui allait être diffusée dans tout le pays par France Culture, la journaliste essaya de recueillir le mythe à l'origine de la danse des masques, mais son interlocuteur wauja refusa de révéler l'information ${ }^{19}$. Puisqu'il était évident qu'il évitait la question, la journaliste insista, poussant le traducteur à mettre en lumière les fondements «mythiques » de la musique exécutée pendant les danses des masques. On lui expliqua que c'était l'absence d'autorisation qui motivait la réticence à révéler le mythe de l'Atujuwá. La journaliste changea alors de tactique et demanda quel était le contexte de la musique de l'événement, affirmant que ça passait «au-dessus des têtes » des Français et qu'ils avaient besoin d'une explication pour pouvoir y comprendre quelque chose. Le Wauja répondit en se référant au premier chant du programme de l'événement montpelliérain, qui venait d'un autre contexte et avait été placé au début du spectacle à un certain point des répétitions.

«Ce chant, nous ne l'avons pas inventé. Vous voyez, ce chant que l'on joue [avec la flûte $[k a w o k a ́(t a ̃ i)]^{20}$, c'est le sanglot de yapojeneju. La flûte dit que le maître va souffrir et mourir. Pour cette raison on va le jouer, le sanglot de yapojeneju. Yapojeneju est un esprit-monstre. Si vous chantez la musique pour vous-même pendant un moment, yapojeneju va venir. Alors, vous allez la jouer. On raconte qu'il $\mathrm{y}$ a très, très longtemps, les femmes dansaient avec les flûtes. [Les gens] dansaient avec une fille en réclusion. Son visage était dissimulé derrière un cache en paille. Elle chantait du matin au soir. Vous voyez, ça a continué, ce chant, le sanglot de yapojeneju. Ce sanglot de yapojeneju est ponuma, une malédiction. Il provoque le deuil du maître, il maudit le maître. C'est tout. Dis-lui ça, mon neveu ${ }^{21}$. »

Nous voulons suggérer que l'interlocuteur wauja donne des indices de ce mythe non pas simplement pour le raconter, mais pour résumer l'insatisfaction que les exécutants wauja avaient accumulée durant leur séjour. Les Wauja ont choisi de jouer cette musique au début du rituel et, en invoquant le fondement mythique des conséquences de ce choix, le 
locuteur affirme que le sanglot de yapojeneju est une menace sérieuse. Il en fait l'équivalent de l'okaiya, la « lamentation funéraire rituelle ». Ainsi, la musique des flûtes dans le premier morceau du concert devrait être lue comme une lamentation funéraire ou un deuil par anticipation, qui amène l'échec de la célébration. Dans le rituel, le conducteur du chant (le rôle de l'interviewé dans le spectacle) peut choisir d'inverser la direction du pouvoir de l'esprit-monstre. Si un mauvais maitre refuse de la nourriture, du tabac, ou d'autres prestations rituelles, jouer ce chant peut détourner les flèches de l'esprit-monstre vers le maitre, conduisant à une rupture complète des relations sociales.

L'interview elle-même a été considérée comme un échec complet par les journalistes de la radio : ils dirent qu'il s'agissait d'une « interview nihiliste » et qu'ils ne pouvaient guère en utiliser plus que le son de la langue wauja, car les réponses, une fois traduites, leur semblaient une série de répliques hors de propos. Cela venait en partie des spécificités de la traduction du wauja en portugais puis en Français, et des diverses atténuations que l'on fit subir à l'interview, afin de modérer le mécontentement du locuteur, au cours de ces traductions successives. Le traducteur, Yanahin Wauja à cette occasion, invoqua certes la relation entre l'esprit-monstre et l'exécutant, décrivant que le chant « disait » que le maitre allait mourir et soulignant que jouer ce chant provoquerait la mort du maitre. Mais il introduisit immédiatement et explicitement dans le discours un élément qui n'avait pas été exprimé par le locuteur originel : la menace de la mort du maître n'était pas applicable dans ce contexte spatiotemporel. Selon lui, le système total n'était applicable qu'au «village » et au maître qui organise le "grand » rituel, c'est-à-dire le rituel thérapeutique traditionnel des Wauja. Ainsi les conditions de production et d'exécution du rituel sont-elles à la source des tensions dans la reconnaissance de son efficacité.

\section{Le message dans la bouteille de Coca-Cola}

Les Wauja ont remarqué à plusieurs reprises que leur séjour en France s'est déroulé sous le signe de la différence culturelle. Par exemple, lorsqu'ils se plaignaient de la qualité de la nourriture, ils reconnaissaient aussitôt qu'il n'y avait pas grand-chose à faire puisque les Français ont des coutumes et des goûts différents ${ }^{22}$. En ce sens, la quantité de nourriture était mise en avant, car cette inquiétude au sujet de la quantité est également cohérente avec l'estimation de l'efficacité rituelle. Dans l'extrait suivant d'une interview, un exécutant exprime son mécontentement vis-à-vis des organisateurs en affirmant que les esprits-monstres sont en colère. Si l'on considère la manière dont les représentants/ exécutants s'identifient aux esprits-monstres, on doit une fois encore prendre en compte le fait que cet homme ne se contente pas de "se plaindre", mais suggère que l'attitude des organisateurs pourrait sérieusement mettre la célébration en danger.

«C'est pourquoi l'Atujuwá est en colère, l'Atujuwá est en colère. L'Atujuwá ne mange pas et ne boit pas de sodas ${ }^{23}$. Il ne reçoit pas les nourritures qu'il aime bien; il ne mange pas différents fruits. L'Atujuwá est en colère... Maintenant il est en colère. Tu vois, ces hommes peuvent devenir faibles et désorientés, l'Atujuwá peut les mettre en transe, et ils peuvent ne pas se réveiller. La faim fait mal/tue l'Atujuwá, tu vois?»

Dans l'éventail des nourritures que les mécènes français n'offraient pas aux exécutants (et donc à l'Atujuwá), c'est l'absence de boissons gazeuses qui devint réellement un des principaux points de désaccord entre les Wauja et les organisateurs. Pour les Wauja qui 
ne sont pas encore très familiers avec le monde extérieur au village, il faut lire cela à la lumière des prestations en grandes quantités de boisson (sucrée) comme biens d'échange donnés en paiement aux exécutants par les maîtres du rituel pendant la préparation et l'exécution de la danse des masques. Les boissons sucrées sont en général hautement valorisées par les Wauja. Le café, par exemple, est un bien de luxe de plus en plus disponible dans la communauté, préparé avec de grandes quantités de sucre raffiné. Mais les sodas comme le Coca-Cola ou le guaraná sont les boissons préférées des Wauja lorsqu'ils se trouvent à l'extérieur de leur village ${ }^{24}$.

41 Le Coca-Cola et le guaraná sont les boissons appropriées dans le monde des Blancs. Ils sont clairement plus valorisés que l'eau, non seulement parce qu'ils sont sucrés et plus chers, mais aussi parce qu'ils représentent les boissons qu'il est approprié d'offrir à un invité. Offrir du Coca-Cola à quelqu'un équivaut, dans les termes de la culture française, à offrir du vin, et non pas seulement de l'eau, à un invité reçu à dîner. Cependant, un représentant du musée du quai Branly sur place, entendant que les Wauja voulaient boire du Coca-Cola, s'exclama : « Mais c'est mauvais pour leur santé ! II passa complètement à côté de l'idée que, pour les Wauja, c'est précisément désirer et ne pas obtenir l'équivalent urbain d'une boisson sucrée décente qu'ils considéraient à la fois comme une offense et comme mauvais pour leur santéén.

\section{Conclusion}

Les objets culturels peuvent habituellement être exposés soit d'une manière esthétique, soit d'une manière pédagogique, ethnographique. Les présentations esthétiques acceptent la décontextualisation comme un facteur positif et inévitable du commerce de la culture. Les présentations pédagogiques l'évitent en fournissant un exposé adéquat de l'arrière-fond ethnographique. Mais dans le cas de l'exécution d'un rituel caractérisé par des processus de transformation, l'orientation ethnographique est une entreprise bien plus difficile. L'anthropologie peut, selon nous, offrir un juste milieu entre les extrêmes de la décontextualisation esthétique et du détail ethnographique excessif, en fournissant un portrait plus exact du contexte de présentation d'un objet culturel. Il s'agit d'accorder plus d'attention aux postures idéologiques, aux pratiques et aux réactions qui concernent l'organisation, l'importation, l'explication et l'exécution de tels objets. On reconnaît ainsi que les formations culturelles générées par de tels contextes vont elles-mêmes contribuer à un processus interculturel qui permet aux Wauja et aux autres peuples indigènes d'occuper de nouveaux espaces culturels et politiques.

43 C'est l'interruption du rituel ou de la danse des masques dans le village wauja en mars 2005 qui nous a fait prendre conscience que les rituels ou les célébrations sont des actes politiques qui doivent prendre en compte tous les participants ou tous les publics impliqués. À cette occasion, toutes les personnes présentes étaient réunies contre le vrai danger qui déclenche la danse des masques Atujuwá en tant que forme de cure rituelle. Même si « le spectacle devait continuer » [« the show must go on »], car il fallait finir la peinture des masques afin de les envoyer en France, et même si les Atujuwá « devaient apparaître ensemble » dans l'intérêt des éventuels films à venir, l'exécution du rituel s'arrêta, et si l'état de l'enfant malade avait empiré, elle n'aurait probablement pas continué. Pour les Wauja, c'était une confirmation que les Atujuwá étaient bien présents. Même si l'infusion végétale était prête pour peindre les masques avec le superbe jaune d'or qui se détache sur le rouge et le noir, personnes n'osait initier la tâche. À ce stade, en 
tant qu'organisateur et directeur scientifique responsable du spectacle en France, Barcelos Neto décida d'entamer lui-même la peinture des masques car la possibilité qu'elle ne soit pas achevée était réelle. Viveiros de Castro (1992) a parlé avec éloquence de la réticence des Indiens d'Amazonie à initier les tâches. Dans notre cas, il y avait un facteur aggravant: la tâche elle-même était potentiellement la source d'effets indésirables. Cependant, l'intervention de Barcelos Neto fonctionna, car au moins deux Wauja prirent sur eux le risque de l'aider ${ }^{26}$. Ainsi, les masques furent prêts à la fois pour le tournage qui suivit, et pour le voyage ultérieur en France.

Mais quelles sont les conséquences de cet acte en termes d'authenticité ou d'identification de l'auteur? Est-ce que le musée du quai Branly devrait signaler dans son catalogue qu'un masque de la collection fut partiellement peint par un anthropologue? Est-ce que cela le rendrait moins authentique en tant qu'objet culturel ? Est-ce que cela mettrait en cause la revendication par les Wauja d'en être les auteurs? Même si les anthropologues suivent habituellement, comme nous-mêmes, la procédure de ne pas interférer directement, les décisions d'interférer ou non avec une certaine situation ont toujours fait partie du travail de terrain. Que ces décisions soient judicieuses ou non ne nous concerne pas ici. Tel est notre argument: ironiquement, un récit complètement ethnographique, qui lutte pour être aussi proche que possible du contexte ethnographique d'un objet culturel, devrait prendre en compte justement l'histoire sociale des objets culturels exposés, même si on doit alors en révéler les caractéristiques les plus hybrides. Puisque les objets culturels, et en particulier ceux qui sont désormais disponibles pour le commerce, sont marqués par des médiations denses, il est important de reconnaître qu'une danse des masques amazonienne amenée en France est une célébration rituelle dont les auteurs sont multiples - spécialistes rituels, passeurs culturels indigènes et non indigènes, anthropologues-directeurs, et organisateurs institutionnels - et ont chacun leur propre position idéologique. Mais, selon nous, c'est ce que les masques ont représenté depuis leur apparition. Ils nous ont précisément renvoyé l'image de la manière dont nous les avons regardés, dont nous les avons lus, et des peuples indigènes qui les portent, car nous ne devrions pas supposer que les publics occidentaux ne seraient pas capables de comprendre une présentation des masques Atujuwá comme étant des miroirs monstrueux révélant nos propres visages disgracieux.

Nous voudrions suggérer que la danse des masques est en partie une compétition entre forces politiques à l'intérieur et à l'extérieur du village, qui subsume celles entre le maître/mécène et le représentant/exécutant, entre les vivants et les esprits-monstres, et entre les Wauja et les Blancs. En ce sens, les Wauja sont constamment en train de faire pencher la balance d'un potlatch : ils font monter les enjeux entre maîtres et exécutants et étirent les frontières des contextes rituels afin de transformer les relations sociales. C'est également pour cette raison, selon nous, que les peuples du Xingu ont tout d'abord régionalisé, et maintenant internationalisé, leurs rituels. Nous espérons avoir fourni à présent suffisamment de preuves que les Wauja ont eu en France une expérience engagée et politique, et pas seulement esthétique. Cependant, si l'on tente d'exprimer en un mot leur message à leurs hôtes français, les Wauja représentant les visiteurs étrangers, le résumé de leurs impressions de la société française serait à peu près celui-ci : « Prenez en compte les différences culturelles parmi vous; traitez les étrangers que vous avez accueillis comme de vrais hôtes, en leur donnant au moins de quoi vivre; et soyez généreux. » Quiconque est familier des problèmes que rencontre la société française aujourd'hui devrait admettre que les Wauja ne sont pas loin de la vérité. 

rapport à leurs perceptions de leurs hôtes français, depuis les répétitions jusqu'à la fin du spectacle de danse de masques à Montpellier. Oubliant toutes les difficultés qu'ils avaient rencontrées depuis leur arrivée en France, les Wauja ont donné un spectacle extraordinaire le soir du concert, avec des moments d'improvisation surprenants, par exemple lorsque l'Atujuwá a échappé au contrôle du leader rituel et a refusé d'entrer dans la «maison des hommes» de la scène. Le public s'est levé. L'exécution du rituel ellemême est devenue une partie du potlatch des Wauja. D'autres danseurs Atujuwá ont commencé à interagir avec le public, et pendant un moment il y eut peu de séparation entre les spectateurs et les danseurs, tant était grande la proximité émotionnelle. Les Wauja ont alors rompu de nouveau le protocole et, faisant taire les louanges et les salves d'applaudissements, ont annoncé à toutes les personnes présentes qu'ils voulaient inviter l'organisateur, le "maître rituel», à se présenter sur scène pour qu'ils puissent lui exprimer leur gratitude pour l'invitation à venir en France.

Cette personne n'est jamais venue.

\section{NOTES}

1. Voir aussi Harrison (2000) sur l'objectification de la culture dans la Mélanésie d'avant le contact.

2. La notoriété du Kwarup vient sans aucun doute partiellement de la popularité de la série Xingu, produite par la chaîne de télévision Rede Manchete dans les années 1980, et du film homonyme, Kwarup (1988), réalisé par Ruy Guerra et issu d'un roman d'Antonio Callado.

3. L'un d'entre nous (Fiorini) était dans le PIX à ce moment-là, conduisant un groupe de recherche consacré à l'histoire sociale des Naruvôtu, un groupe caribe qui fut décimé par les épidémies apportées dans la région par l'expédition de Villas-Boas et qui ne put maintenir son statut de groupe local indépendant (voir Fiorini 2005). Comme les Kalapalo (avec qui les Naruvôtu vivent désormais) ont massivement assisté à ce Kwarup, Fiorini y fut invité afin de mettre en évidence le travail du groupe de recherche, à la fois localement et par l'intermédiaire des médias présents.

4. De plus, leur participation ne devrait pas être passée sous silence. Les leaders du PIX ont institué un droit d'entrée pour les anthropologues qui étudient les groupes indigènes de la région depuis un certain nombre d'années déjà.

5. Bien que nous adoptions dans ce qui suit un regard critique sur l'événement qui s'est déroulé en France, puisque nous essayons de présenter la perspective des Wauja, nous tenons à préciser que nous ne tentons pas de dénigrer les efforts de nos collègues, et en particulier d'Aristóteles Barcelos Neto ou des membres du musée du quai Branly qui ont organisé le spectacle de danse wauja à Montpellier. Nous pensons que c'est précisément à travers l'exposition à de tels projets que les Wauja et d'autres groupes indigènes peuvent établir leurs critères pour négocier - dans leurs propres termes - la présentation de leurs objets culturels.

6. Nous adoptons ici la traduction d'« esprit-monstre " pour le terme wauja apapaatai, en partie en réponse aux questions de conseillers linguistiques wauja qui ont demandé à Christopher Ball pourquoi ne pas désigner apapaatai par le portugais monstros, "monstres", ce qui était 
probablement motivé par leur désir d'indiquer les effets potentiellement nuisibles du contact humain avec ces esprits. Ainsi, dans la traduction hybride d' " esprit-monstre », « esprit » renvoie aux conditions ontologiques des apapaatai comme personnes non humaines et sujets intentionnels [agentive], alors que «monstres » souligne le risque potentiel qui découle de leur proximité avec les humains, et qui se manifeste dans la maladie. Voir aussi Basso (1973) sur les notions kalapalo de itseke comme des « monstres".

7. Kawoká renvoie à l'esprit-protecteur, et le modificateur -mona dénote une instance de l'espritprotecteur, ou quelque chose qui y ressemble.

8. L'argent en espèces est également important, mais n'a pas (encore) remplacé complètement les autres formes de paiement.

9. Steinen a observé un grand masque chez les Bakairi, le tüwetüwe, dont la base était une large jupe de paille, et dont la danse était faite de tourbillons et de balancements. L'extrémité supérieure de ces masques était cependant beaucoup plus petite que celle des masques Atujuwá des Wauja contemporains. Curieusement, les Bakairi qui ont montré ce masque à Steinen ont apparemment tenu à lui préciser qu'il ne s'agissait pas d'une représentation des Bakairi (1940 [1894] : 393).

10. Sur la fascination des Indiens du Xingu pour les chapeaux de l'expédition de 1884, voir Steinen (1942 [1887] : 233). Le même passage pourrait contenir une explication de l'idée que les Blancs remplissaient l'air de leur danger et de leur pouvoir, car l'expédition fit souvent feu sur les Trumai.

11. Fiorini a été invité à filmer l'événement dans le cadre d'un projet toujours en cours dans le village wauja, dont le but est d'enseigner la photographie et la vidéo numérique aux membres de la communauté. Le principal négociateur indigène de la danse des masques exécutée en mars 2005 comme du spectacle réalisé en France, Yanahin Wauja, est en fait un cinéaste partenaire dans ce projet. Il a lui-même photographié et filmé ces deux événements dans le but de réaliser un film documentaire en partenariat.

12. Ces deux situations étaient décrites comme indésirables pour l'individu en question.

13. Christopher Ball remédia en partie à cette situation en prévenant Marcelo Fiorini, lorsque ce dernier arriva à Canarana, qu'il n'y avait plus de tabac dans le village wauja. Le lendemain matin, ils en achetèrent tous deux un stock copieux, qui fut ensuite emporté dans le village. Les Wauja considèrent en effet le tabac comme un substitut lorsque la nourriture fait défaut.

14. Dans le Haut-Xingu, l'escargot a un équivalent aquatique dont on utilise les coquilles pour fabriquer les colliers, mais qui est classé comme incomestible. Le problème des portions a été partiellement résolu par l'embauche d'une équipe de restauration chargée de nourrir le groupe lors des répétitions, ce qui permit aux Wauja de manger plus copieusement.

15. Christopher Ball a obtenu ces témoignages auprès des exécutants wauja pendant leur séjour d'une semaine en France. Ces textes ne représentent pas des digressions aberrantes ou forcées sur le sujet de l'insatisfaction, car le groupe en parlait fréquemment.

16. Ici, l'usage de «faire » renvoie à la construction du rituel qui amène la coprésence avec l' Atujuwá. Dans sa première affirmation, le locuteur introduit la réalité ontologique basique de l' Atujuwá. Ceci présuppose une prise en considération du fait que d'autres, les Français par exemple, pouvaient ne pas partager ses vues.

17. Le locuteur invoque l'état dans lequel il a vu l'Atujuwá - un rêve, sans le mentionner explicitement - car il s'agit d'un principe culturel admis.

18. Ce fut donc à d'autres personnes que les organisateurs d'acheter du tabac pendant la phase de production, ainsi que du tabac et du Coca-Cola pour les hommes durant leur séjour en France.

19. L'interlocuteur wauja, un des principaux organisateurs de la danse, qui jouait ici le rôle d'un représentant de kawoká-mona, était un locuteur virtuellement monolingue, avait un savoir rituel spécialisé, et était chargé de conduire la célébration. Il prétendit cependant être ignorant du mythe, disant : «Je ne sais pas, je suis encore trop jeune. » 
20. Les flûtes kawoká sont des flûtes sacrées conservées hors de la vue des femmes, qui sont des emblèmes culturels célèbres en Amazonie. Dans ce contexte, le chant est normalement joué sur des flûtes kawoká. Lors du voyage en France, cependant, les grandes flûtes kawoká ne furent pas autorisées à quitter la communauté parce qu'elles étaient trop «chères » ou valorisées, et la musique fut jouée sur de plus petites flûtes appelées kawokàtã.

21. Le locuteur invoque ici une référence spatiotemporelle « mythique ", une époque où il n'était pas interdit aux femmes de voir les flûtes kawoká, afin d'expliquer que yapojeneju, un espritmonstre féminin associé avec la forêt, possède un chant plaintif qui provoquera la souffrance et la mort du maître lorsqu'il est joué avec les flûtes. Ceci est clairement opposé à " aujourd'hui », où les sanctions en cas de violation de cette règle de la part des femmes sont sévères.

22. Tous les artistes wauja ne dirent pas qu'ils avaient faim en France. Un jeune homme qui ne fumait pas de tabac, par exemple, affirma que, dans le village, les gens ne mangent pas régulièrement, et parfois pas du tout de la journée. Il dit que c'était bon pour lui de manger moins car il était «au régime » et ne voulait pas avoir " un gros ventre ». Il dit aussi que les Indiens pouvaient supporter la faim. Ces affirmations elles-mêmes semblent confirmer les plaintes des autres.

23. La boisson mentionnée ici est en fait le guaraná, un soda qui est l'emblème du Brésil comme le Coca-Cola est celui des États-Unis. En portugais courant, « guaraná » représente aussi un terme général pour « soda », comme " coke » en anglais américain.

24. Les Wauja nous ont dit à Montpellier qu'ils mélangeaient des sachets de sucre pris à la cafétéria aux réserves d'eau minérale en bouteille que leur avaient données les organisateurs (et ainsi sembla se résoudre, de leur point de vue, la question de la salinité de l'eau potable).

25. Le jour de la célébration, les Wauja découvrirent à la cafétéria de l'université qu'un verre de Coca-Cola ou d'un autre soda était inclus dans leurs tickets-repas. Ils étaient si contents que les employés à la caisse leur dirent qu'ils pouvaient avoir autant de coca qu'ils voulaient - et les Wauja s'attachèrent donc à vider les deux machines à boisson.

26. Un autre facteur peut avoir conduit les Wauja à aider l'anthropologue dans ce cas: ils prennent très au sérieux la peinture des masques, comme une composante intégrale de l'exécution du rituel, et voir un étranger peindre les masques a peut-être été hors de question pour certains d'entre eux. Nous remercions Maria Ignês Mello d'avoir souligné cette possibilité.

\section{AUTEURS}

\section{MARCELO FIORINI}

Chercheur invité, CNRS, EREA, Université de Paris X-Nanterre, fioriniorama@gmail.com

\section{CHRISTOPHER BALL}

Doctorant, programme doctoral commun des départements d'anthropologie et de linguistique, Université de Chicago, cgball@uchicago.edu 